\title{
EN BUSCA DE LA EVALUACIÓN DE LA EDUCACIÓN SUPERIOR EN URUGUAY: UN CASO DE ADOPCIÓN TARDÍA Y ASIMETRÍA ESTRUCTURAL
}

\author{
IN SEARCH OF URUGUAYAN HIGHER EDUCATION QUALITY ASSURANCE: \\ A CASE OF LATE ADOPTION AND STRUCTURAL ASSIMETRY
}

\author{
EM BUSCA DA AVALIAÇÃO DO ENSINO SUPERIOR NO URUGUAI: \\ UM CASO DE ADOÇÃO TARDIA E ASSIMETRIA ESTRUTURAL
}

\begin{abstract}
Enrique Martinez Larrechea ${ }^{1}$ Adriana María Chiancone Castro ${ }^{2}$

\section{RESUMEN}

El texto analiza el caso de las políticas de evaluación de la educación superior en Uruguay, único país del MERCOSUR que no generó una agencia de acreditación nacional ni tampoco regional y cuyo debate público sobre evaluación posee características muy especiales. El trabajo presenta el caso uruguayo en el contexto de los países de la región y describe e identifica las principales iniciativas relativas a la evaluación, la acreditación y el aseguramiento de la calidad en el país. Este sector de las políticas públicas aparece cruzado por dos dimensiones claves para comprender el caso nacional: en primer lugar, una fortísima asimetría estructural del sistema de educación superior, en proceso de transición hacia nuevos escenarios. En segundo lugar, su condición de posible late adopter, o adoptante tardío de estas políticas, genera especiales desafíos para el país, que difieren de los que enfrentaron los países que generaron tempranamente políticas públicas bien definidas. La discusión final de las iniciativas y debates en curso permite concluir que las políticas públicas de evaluación y la acreditación de la educación superior son productos históricos concretos, que no pueden trasladarse acríticamente de un país a otro, pero tampoco de un tiempo histórico a otro, marcado por una aceleración de las tendencias globales y locales de transformación. En tales condiciones, se requiere una cierta dosis de imaginación crítica y el texto aporta categorías conceptuales relevantes para asumir la tarea de construir una cultura de la evaluación en la educación superior uruguaya.
\end{abstract}

PALABRAS-CLAVE: Políticas públicas. Evaluación. Acreditación. Aseguramiento de la calidad. Educación superior. Educación comparada.

\begin{abstract}
The chapter analyzes the case of higher education evaluation policies in Uruguay, the only country within MERCOSUR which did not generate neither a national nor a regional accrediting agency and whose public debate on evaluation has very special features.

The paper presents the case of Uruguay, in the context of the countries of the region and describes and identifies the major initiatives on evaluation, accreditation and quality assurance in the country. This sector of public policy appears crossed by two key dimensions to understand the domestic case: first a very strong structural asymmetry of the higher education system in transition to new scenarios. Second, his condition of late adopter raises special challenges for the country, which differ from those faced by all those countries where defined public policies have been adopted. The final discussion of the initiatives and ongoing discussion leads to the conclusion that public policy evaluation and accreditation of higher education are concrete and historical products, they can not be transferred uncritically from one country to another, neither from a historical period to another, especially one of acceleration of global and local trends of transformation. In such conditions, a certain amount of critical imagination is required and the relevant chapter provides conceptual categories to take on the task of building a culture of evaluation in the Uruguayan higher education.
\end{abstract}

\footnotetext{
${ }^{1}$ Doutor em Relações Internacionais. Mestre em Politicas Educativas; Reitor do IUSUR; Presidente da Sociedade Uruguaia de Educação Comparada e Internacional; Pesquisador -SNI. E-mail: martinez.larrechea@gmail.com

2 Profesora del Instituto Universitario Centro Latinoamericano de Economia Humana. E-mail: achiancouniversidad@gmail.com

Submetido em: 08/11/2016 - Aceito em: 14/12/2016.
} 
KEYWORDS: Public policies. Evaluation. Accreditation. Quality assurance. Higher education. Comparative education life histories.

\section{RESUMO}

O texto analisa o caso das políticas de avaliação da educação superior no Uruguai, o único país do Mercosul que não gerou uma agência de acreditação nacional, ou regional, e cujo debate sobre avaliação tem características muito especiais. O trabalho apresenta o caso do Uruguai no contexto dos países da região e descreve e identifica as principais iniciativas sobre avaliação, acreditação e garantia de qualidade da educação superior no país. Este setor de política pública aparece atravessado por duas dimensões-chave para entender o processo nacional: primeiro uma forte assimetria estrutural do sistema de ensino superior na transição para novos cenários. Em segundo lugar, a sua condição de adoção tardia dessas políticas suscita desafios especiais para o país, que diferem daqueles enfrentados por todos os países que possuem políticas públicas definidas. A discussão final sobre as iniciativas e debates em curso leva à conclusão de que a avaliação de políticas públicas e acreditação do ensino superior são produtos históricos concretos, eles não podem ser transferidos de forma acrítica de um país para outro, tampouco de um período histórico para outro, em especial num contexto marcado por uma aceleração das tendências globais e locais de transformação. Em tais condições, é necessária uma reflexão crítica e o texto fornece categorias conceituais que contribuem para a construção de uma cultura de avaliação no ensino superior uruguaio.

PAlaVRAS CHAVE: Políticas públicas. Avaliação. Acreditação. Garantia da qualidade. Educação superior. Educação comparada.

\section{En Busca de la Evaluación de la Educación Superior en Uruguay: Un Caso de Adopción Tardía y Asimetría Estructural}

El sistema de educación superior en Uruguay, es el único del MERCOSUR que no generó a lo largo de los últimos veinte años, una agencia nacional de evaluación o de acreditación nacional o regional, como fue la tendencia general en América Latina.

Las principales iniciativas en este campo estuvieron limitadas a un ambiente sectorial de desarrollo o no contaron con el consenso de los principales actores para llegar a traducirse en políticas públicas.

El país carece no solo de un "libro blanco" o de una "blueprint" para enmarcar algún posible proceso de desarrollo, sino que no existen alternativas de política disponibles, a excepción del proyecto gubernamental de creación de una Agencia específica, que aun reformulada de un período de gobierno a otro, fue retenida en el Poder Legislativo a lo largo de ya siete años, reflejando la perplejidad del sistema de educación superior uruguayo en lo relativo al aseguramiento de la calidad.

El trabajo presenta el caso uruguayo en el contexto regional, analiza el proceso cumplido desde 1995 y el estado de situación actual, describiendo asimismo las principales iniciativas relativas a la evaluación, la acreditación y el aseguramiento de la calidad en el país y las características del debate público sobre la evaluación y la acreditación universitarias.

Estas posibles políticas públicas aparecen cruzadas por dos dimensiones claves para comprender el caso nacional: en primer lugar, una fortísima asimetría estructural del sistema 
de educación superior, en proceso de transición hacia nuevos escenarios. En segundo lugar, su condición de posible late adopter, o adoptante tardío de estas políticas, genera especiales desafíos para el país, que difieren de los que enfrentaron los países que generaron políticas públicas definidas ya desde finales de la década de 1980 o durante la de 1990.

En tales condiciones, se requiere una cierta dosis de imaginación crítica y el texto intenta aportar categorías conceptuales relevantes y posibles alternativas de políticas públicas, generadas en el ámbito académico, que podrían permitir el asumir la tarea de construir una cultura de la evaluación en la educación superior uruguaya.

La discusión final de las iniciativas y debates en curso permite concluir en que las políticas públicas de evaluación y la acreditación de la educación superior son productos históricos concretos, que no pueden trasladarse acríticamente de un país a otro, pero tampoco de un tiempo histórico a otro, en especial en una fase caracterizada por una aceleración de las tendencias globales y locales de transformación.

\section{El -tardío- ingreso del tema en la agenda pública}

El tema de la calidad de la educación superior ingresó en la agenda pública uruguaya, tardíamente, a mediados de la década de los años noventa, como resultado de la reinstitucionalización del país, del restablecimiento de la autonomía universitaria en 1985 y del segundo censo universitario de 1988, que cuantificó el notable incremento de la matrícula experimentado entre 1974 y 1988, el que pasó de menos de veinte mil a más de sesenta mil estudiantes.

Adicionalmente, el sector privado comenzó a estructurarse en base al decreto-ley que había autorizado el funcionamiento de la Universidad Católica. Otras instituciones requirieron también una reglamentación que permitiese seguir el mismo procedimiento acordado a dicha universidad.

En 1995, el decreto $\mathrm{N}^{\circ} 308 / 995$ estableció un sistema de reconocimiento de condiciones iniciales para el funcionamiento de instituciones universitarias privadas y en los años siguientes, otras tres universidades privadas fueron reconocidas.

1997 y 19988 resultaron dos años clave, por dos razones. En primer lugar, porque la Universidad de la República conformó una Comisión de Evaluación Institucional encargada de los procesos de auto-evaluación y evaluación institucional de sus facultades, áreas y servicios, en base a partir de documentos de trabajo relativos a un documento de Lineamientos y Criterios para la Autoevaluación y Evaluación. El primer ejercicio de este tipo fue la realización de un proceso de evaluación en el Área Tecnológica - conformado por las facultades de Ingeniería, Arquitectura y Química. 
A lo largo de una década, se cumplieron diversos procesos de autoevaluación y evaluación institucional de facultades. Sin embargo, una década y media más tarde, es poco lo que se sabe del posible impacto de estos ejercicios, que tuvieron apenas un ciclo por servicio. Poco se sabe sobre cuanto aportaron a la construcción de una cultura de la calidad.

Otros ejercicios se focalizaron en el Programa de Desarrollo de Ciencias Básicas, PEDECIBA, cogestionado entre UDELAR y el MEC, y en el Plan Estratégico de la UDELAR, el PLEDUR.

La Comisión de Evaluación Institucional de UdelaR, es un tipo de política emparentada con la experiencia desarrollada por el Programa de Auto-avaliação das Instituções e Universidades Brasileiras -PAIUB-, con un fuerte énfasis en la evaluación como proceso autónomo e la comunidad universitaria, en lo educativo y en lo político y autónomo. Es decir, fue diseñado con el fin de cumplir un rol anticipatorio a la posible elaboración de políticas gubernamentales de quality assurance, como en Argentina.

En segundo lugar, en el marco del Sector Educativo del MERCOSUR, tuvo lugar en 1998 la firma de un Memorándum de Entendimiento por parte de los Ministros de Educación del MERCOSUR destinado a establecer un Mecanismo Experimental de Acreditación de carreras universitarias de grado.

En los años siguientes tuvo lugar la construcción de dimensiones, criterios e indicadores por parte de Grupos de Expertos, antes de que la Reunión de Agencias Nacionales de Acreditación -RANA- y el Sector Educativo del MERCOSUR desarrollaran una operación de pre-test, realizado en 2002 (MARTIN, 2000).

A partir de 2003 fue posible aplicar la metodología diseñada para el Mecanismo Experimental de Acreditación -MEXA.

El Memorándum de Entendimiento requería la constitución de Agencias nacionales de Acreditación en cada país, si bien la institucionalidad difería en cada una de ellas, podía considerarse que Argentina, con la CONEAU, Brasil, con CAPES e INEP y Chile con la Comisión Nacional de Acreditación de Pregrado, satisfacían ese requerimiento, mientras que Paraguay y Uruguay carecían por el momento de tales agencias. En enero de 2003 Paraguay creó la ANEAES.

Uruguay pudo participar en los mecanismos regionales de acreditación (primero MEXA, y luego el mecanismo permanente de Acreditación de Carreras Universitarias de grado -ARCUSUR/ARCUSUL) a través de una Comisión ad hoc de Acreditación (DÁVILA; MARTÍNEZ LARRECHEA, 2009).

La política anticipatoria de la Universidad de la República, el decreto regulatorio de la autorización de universidades privadas y la firma del Memorándum de Entendimiento, fueron 
tres factores de promoción del debate en torno a las necesarias políticas de aseguramiento de la calidad. En especial, la necesidad de participación en el mecanismo experimental resultaba un factor sistémico, que interpelaba a ambos subsectores, público y privado.

Sin embargo, pese al impulso dado por estos factores, no fue posible un consenso nacional sobre la política de aseguramiento de la calidad de la educación superior. La crisis del 2002 y la rotación de partidos en el gobierno, a partir de 2005, generaron las condiciones para una relativa vuelta a cero de la agenda.

La evaluación de la calidad no consiguió ingresar en la agenda pública, a partir de políticas gubernamentales orientadas a la accountability, o de reelaboración del vínculo entre estado y educación superior, sino de forma mediada por el cambio de orientación política, en un contexto pos crisis.

\section{El proyecto de creación de la APACET}

El proceso de aprendizaje realizado por el sistema uruguayo, se sustentó en tes factores: i) la evaluación institucional del Área Tecnológica de la UDELAR, y la posterior extensión de este proceso a diversas facultades ${ }^{2}$; ii) algunas experiencias privadas y, sobre todo, iii) el Memorándum de Entendimiento de los Ministros del MERCOSUR (1998) y el posterior proceso del MEXA y primera etapa de ARCUSUR.

Este proceso de aprendizaje y experimentación no se resolvió positivamente en la creación de una institucionalidad consensuada -salvo la Comisión Ad Hoc de Acreditación creada para hacer frente a las exigencias inmediatas de los ciclos de MEXA y ARCUSUR.

Las diferencias entre el sector público y el sector privado respecto al peso relativo que cada uno de ellos debería tener en la posible agencia, ocluyeron la posibilidad de un consenso. En la Universidad de la República, algunos sectores reclamaban ejercer la regulación del sector privado, al que percibían como ilegítimo; mientras que, en el sector privado, algunos se negaban a cualquier tipo de regulación estatal. Ambos extremos bloquearon posibles entendimientos de los núcleos de técnicos e investigadores que poseían experiencia concreta -de algún tipo- en los procesos de aprendizaje institucional y colectivo antes mencionados.

Incluso un borrador que sugería la posibilidad de desarrollar las funciones de autoevaluación y evaluación institucional y de programas, en forma voluntaria, sin consecuencias públicas, a partir de una Fundación que permitiera sistematizar la experiencia del sistema, cayó en el vacío.

\footnotetext{
${ }^{2}$ Sucesivamente en el Decanato de Ingeniería y en el Rectorado del Ingeniero Rafael Guarga \begin{tabular}{l|l|l|l|l|l|l} 
(C) Rev. Inter. Educ. Sup. & Campinas, SP & v.2 & n.3 & p.573-586 & set./dez. 2016 & ISSN 2446-9424
\end{tabular}
} 
A partir de 2005, el Ministerio de Educación y Cultura elaboró un proyecto de ley de creación de una Agencia de Promoción y Acreditación de la Calidad de la Educación Superior. Inicialmente presentado en 2007 , el proyecto recibió críticas que postergaron su tratamiento. La reiteración del mismo en 2010 tuvo el mismo destino, en la medida en que las características del mecanismo propuesto, se sustentaban en una lógica "top-down" de formulación de políticas, pese al funcionamiento previo de una comisión de redacción integrada por miembros designados ad hoc por el Ministerio. El proyecto dejaba fuera cualquier acción efectiva de promoción de la calidad -cuyos mecanismos normales deberían haber sido la auto-evaluación y la evaluación institucional para la mejora- y se centraba en cambio en mecanismos sumativos, como la inspección administrativa y la acreditación.

Los mecanismos inspectivos que se proponían pretendían complementarse con una lógica de pares, pero en ausencia de estándares consistentes y consensuados que permitieran sustentar un juicio público, la misma acreditación resultó privada de fundamentos.

El único incentivo para la acreditación voluntaria consistía en evitar la directa intervención inspectiva, pero no se traducía en ningún efecto que ampliara el espacio de autonomía de las universidades e institutos privados.

Además, el proyecto creaba dos circuitos diferenciales, uno para la Universidad de la República y otro para el sector privado, fragmentando y debilitando, en vez de promoverla, la cultura de la evaluación y la lógica de la calidad.

De esta manera, el proceso de aprendizaje previo no pudo ser sistematizado, no recibió el respaldo de una institucionalidad reforzada y en cambio se produjo un desdibujamiento de la cuestión e incluso su desaparición de la agenda pública a partir de 2010 .

\section{La matriz tradicional y la prórroga estructural de las reformas}

El estado de bloqueo que presentan las políticas públicas en este tema, reflejan las tensiones que se producen entre la matriz inercial de la educación superior, que se traduce en lo que hemos llamado "la prórroga estructural de las reformas" (MARTÍNEZ LARRECHEA; CHIANCONE, 2006) y los nuevos y fluidos escenarios de innovación e internacionalización, por los que transita la región del MERCOSUR.

La "prórroga estructural de las reformas" es un fenómeno por el cual los actores sociales y la estructura del sistema convergen para demorar la adopción de cambios, o para neutralizarlos una vez adoptados.

En efecto, Brasil produjo aproximadamente en la misma época -las últimas dos décadas y media- un conjunto impresionante de políticas públicas de alto impacto - 
eventualmente contradictorias entre sí- que transformaron la faz de la educación superior. Merecen citarse al respecto el Programa de Autoavaliação de Instituições e Universidades do Brasil -PAIUB; el Examen Nacional de Cursos -ENC-Provão; la creación del Sistema Nacional do Ensino Superior -SINAES (2004); y la posterior actuación del MEC, a través del Concepto Preliminar de Curso y el Índice General de Cursos (2008). Merecen citarse también las medidas de democratización del acceso a la educación superior, como el Fondo de Financiamiento de Estudiantes de la Educación Superior -FIES (2001) y el programa ProUni (2005), así como, en materia de calidad e internacionalización, el programa Ciencia sin Fronteras. Adicionalmente, Brasil generó nuevas universidades públicas, dotadas unas de alta pertinencia local y otras orientadas a la integración regional y desarrolló la Universdidade Aberta do Brasil. Todo lo cual indica, más allá de los debates domésticos, el dinamismo de las políticas públicas y la respuesta, relativamente convergente, del sistema (GARCIA DOS REIS, 2011). Lo mismo puede decirse de Argentina, que creó la Secretaría de Políticas Universitarias, el Programa de Incentivos para Docentes-Investigadores -PROINCE- y el Fondo de Mejoramiento de la Calidad de la Educación Superior -FOMEC; desarrolló una primera experiencia de acreditación de posgrados (CAP) y creó la Comisión Nacional de Evaluación y Acreditación Universitaria - CONEAU, desarrollando al mismo tiempo un exitoso proceso de diferenciación institucional, con la creación de numerosas universidades públicas y privadas (SUASNÁBAR; ROVELLI, 2011, p. 28 y 29).

Paraguay creó hace más de una década, su Agencia Nacional de Evaluación y Acreditación de la Educación Superior (ANEAES). Más recientemente, fue aprobada una nueva ley de educación superior que seguramente habrá de ser el marco para la necesaria expansión de la matrícula.

Todo ello contrasta con la capacidad de la educación superior uruguaya para prorrogar todo lo posible los rasgos básicos de su estructura, alterada solo por innovaciones incrementalistas y muy pocas de tipo estructural. Entre estas últimas, hay que citar la creación del Fondo de Solidaridad Universitaria 81994), la creación de la Facultad de Ciencias (1990), la constitución de la Comisión Sectorial de Investigación Científica de UdelaR (1990) y luego de un Sistema Nacional de Investigadores -SNI- y de una Agencia Nacional de Investigación e Innovación (2006).

La Agencia Nacional de Investigación e Innovación (ANII) fue un aporte novedoso, en tanto conformó una institución nacional, por encima de la lógica dualista UdelaR / Sector privado. De este modo, se conformó como una agencia con cierta dosis de racionalidad weberiana, apta para generar un esquema estratégico diferente. Aunque formalmente dependiente del Gabinete Ministerial para la Innovación, y de un Equipo interministerial, la ANII, creada a fines de 2006, constituye una instancia nacional que sumó a la implementación, la formulación de políticas públicas, relativas a apoyar con planes, programas e instrumentos el desarrollo científico y tecnológico y a fortalecer las capacidades de innovación. 
Más recientemente, se quebró formalmente el monopolio universitario público de la UDELAR con la creación de la nueva Universidad Tecnológica, pero la muy gradual y débil implantación de nuevas ofertas de esta última, de hecho, convalida aquel viejo monopolio, que solo la efectiva constitución de una Universidad de Educación podría alterar (universitarización del sistema de formación docente que aún no aparece con nitidez en la agenda pública).

Estas fueron sin duda las modificaciones sistémicas de las últimas dos décadas, pero resultan insuficientes para dar cuenta de los déficits acumulados y en particular de la falta -ya crónica y persistente, con vocación de rasgo estructural- de un sistema de aseguramiento de la calidad.

\section{Posibles políticas alternativas}

En este contexto, ¿cuáles podrían ser las alternativas de política para la creación efectiva y sustentable de un sistema de aseguramiento de la calidad de la educación superior?

¿Vale la pena insistir en respuestas dadas en contextos de pos-crisis de 2002 en situación de virtual monopolio público de la educación superior ejercido por una sola institución y cuando el sistema de ordenamiento del sector privado tenía apenas diez o doce años?

Todas estas realidades se alteraron básicamente y los desafíos configuratorios y regulatorios del sistema de educación superior se han incrementado, con la presencia regional de grandes conglomerados privados "for profit" (aún no operativos en el país) y con la creciente presencia de proveedores internacionales (esto último sí un fenómeno ya experimentado, sea a través de presencia comercial o de la oferta de formación deslocalizada y a distancia).

El punto central del bloqueo en la construcción de un sistema válido de aseguramiento de la calidad reside en que no ha existido una conceptualización adecuada de los niveles, propósitos, focos, e instrumentos alternativos de un sistema de aseguramiento de la calidad.

Se ha puesto el foco en la acreditación como elemento ordenador del sistema, olvidando que la acreditación requiere un juicio público, y que ese juicio público solo puede ser emitido en relación con un estándar, con un valor consensuado en las diversas dimensiones, criterios e indicadores del sistema. Un consenso público, generalizado en las instituciones y en los actores del sistema requiere de un previo trabajo colectivo en torno a una perspectiva conjunta y, a menudo, este tipo de consenso se cristaliza en una ley.

Pero la acreditación no es el único mecanismo en un sistema de aseguramiento de la calidad y definitivamente no es el primer paso en la construcción del mismo. Es más bien un 
punto de llegada, a partir de reconocer la presencia de instituciones y proyectos académicos diversos, que participan de una cultura de la calidad y que desarrollan con marcada autonomía relativa sus propias estrategias de desarrollo académico y de autoevaluación de las posibles formas de calidad resultantes.

Dos tradiciones resultan relevantes para las estrategias de aseguramiento de la calidad en América Latina: una es la tradición norteamericana, históricamente preceptiva, de carácter regional y fuertemente volcada a la acreditación institucional; otra es la europea, que tradicionalmente rehuyó la acreditación y se basó en un equilibrio entre el control estatal y la evaluación académica basada en la mejora, sin juicios públicos de acreditación (LEMAITRE: ZENTENO, 2012; 48).

El Informe de CINDA 2012 muestra que ambas tradiciones han evolucionado hacia una perspectiva más integrada: en los Estados Unidos, la acreditación se complementa con otras estrategias (licenciamiento, certificación) y con mayor atención a la auditoría académica. Asimismo, en Europa, la última década y media ha visto el surgimiento de esfuerzos por la construcción de sistemas de garantía de la calidad.

Esto es, las décadas de los noventa y dos mil, en América Latina, y de dos mil en Europa nos mostraron una tendencia firme de creación de agencias nacionales y regionales de evaluación y acreditación, así como la tendencia a llevar la garantía de la calidad a nivel de las agencias.

Asimismo, la acreditación institucional se percibe como un mecanismo importante en términos de reducción de la complejidad de gestión de los sistemas de aseguramiento de la calidad y como estrategia de reducción de los costos multiplicados de la acreditación de programas. La acreditación institucional permite revisar dimensiones de calidad que poseen una influencia cierta en la calidad de los programas. Sin embargo, la acreditación institucional no permite dar cuentas de aportes que solo puede realizar la acreditación de programas, en términos de "accountability" y satisfacción de los interesados clave y también en términos de reconocimiento de diplomas (LEMAITRE; ZENTENO, 2012, p. 48-51).

Lemaitre y Zenteno (2012, p. 50-51) nos recuerdan que el aseguramiento de la calidad es más bien un medio que un fin y que es necesaria: "una constante revisión del concepto de calidad y su operacionalización (...)" y que es necesario "definir y aplicar estándares diferentes a distintos programas o instituciones". Según estas autoras "los criterios demasiado prescriptivos generan una cultura de la obediencia". Para promover la calidad los organismos de aseguramiento de la calidad deben" 'desaprender' aquello que hasta ayer era eficaz, pero hoy dejó de serlo.

En este contexto de desarrollo de sistemas más complejos e integrados de aseguramiento de la calidad, con mayor versatilidad conceptual y metodológica, resulta de enorme interés algunas experiencias en el contexto del aseguramiento de la calidad en

\begin{tabular}{l|l|l|l|l|l|l} 
(C) Rev. Inter. Educ. Sup. & Campinas, SP & v.2 & n.3 & p.573-586 & set./dez. 2016 & ISSN 2446-9424
\end{tabular}


Europa. En particular, los llamados "Criterios y Directrices para la Garantía de la Calidad en el Espacio Europeo de Educación Superior” (European Standards and Guidelines), que denominaremos en adelante ESG, aportan una novedad conceptual y metodológica relevante, ordenadora de la acción de las agencias europeas.

En especial porque estas ESG, al decir de Guy Haug (2012, p. 81): “confirman el papel fundamental que tiene cada universidad para garantizar y mejorar la calidad de sus propios programas de estudios".

Esta responsabilidad de las instituciones, se expresa en los llamados Sistemas Internos de Garantía de la Calidad -SIGC. De este modo, gran parte del sistema de aseguramiento de la calidad reside en la "auditoría externa de calidad del SIGC" de las instituciones, una tendencia apreciable en países como Alemania, Austria y Suiza. Al decir de Haug (2012, p. 88-89) "se oyen por todas partes críticas por el exceso de burocracia, los riesgos de un conformismo formal y superficial en vez de auténtico fomento de la calidad" y en esa medida: una respuesta por lo menos parcial a esas críticas se puede notar en el campo de la acreditación, en el movimiento en dirección hacia evaluaciones más estratégicas, y más respetuosas de la diversidad y la autonomía de las universidades, con base en el reforzamiento de sus SIGC. Estos SIGC y la gobernanza universitaria misma, son considerados como "los únicos garantes efectivos de la calidad, la relevancia y la innovación continua". En la estrategia europea esto debería acompañarse de sistemas externos de garantía de la calidad más transparentes, en una revisión de las ESG, en el desarrollo de "sellos de calidad" regionales, en mayor atención a la dimensión externa y a la visibilidad externa y en un sistema de rankings más multidimensionales y pertinentes (HAUG, 2012, p. 89).

Desde luego, una perspectiva de aseguramiento de la calidad que enfatice la autonomía académica, el foco en el mejoramiento y en la cultura de la calidad, tiene sólidos anclajes en la mejor literatura latinoamericana sobre calidad y aseguramiento de la calidad de la educación superior (KELLS, 1993; DIAS SOBRINHO, 2010; SANTOS FILHO, 2016).

Así, parece que las alternativas pasan por la adopción consensuada de un esquema de autorregulación, promovido -aunque no sea exclusivamente- con fondos públicos, que permitan desarrollar la cultura de la calidad de las instituciones. El principal dispositivo de un esquema de autorregulación, parece ser la promoción y fortalecimiento de mecanismos de garantía interna de la calidad; es decir del auto-estudio, la autorregulación, el foco en la mejora continua y la puesta en valor de proyectos institucionales y académicos distintivos. La perspectiva externa, la evaluación y acreditación y el juicio público basado en estándares , un sistema que el Uruguay no ha podido transformar a lo largo de diversos ciclos y en la sucesión de muy diversas coaliciones de gobierno, sin desaparecer por completo de la agenda pública, debería reformularse como un sistema orientado a fortalecer -promover realmente, no como maquillaje del control heterónomo- para todas las instituciones, potentes sistemas internos de garantía de la calidad, en línea con los ejercicios de evaluación externa. 


\section{Discusión}

Como se ha tenido oportunidad de advertir, el sistema de educación superior de Uruguay se enfrenta pues a un desafío clave: dejar atrás la lógica emergente e inercial de transformación y asumir un esquema concreto, pero flexible, de políticas públicas interconectadas y de alto impacto (CHIANCONE; MARTÍNEZ LARRECHEA, 2011; DÁVILA et. al, 2014).

Como hemos tenido oportunidad de sostener, la "matriz tradicional" del sistema de educación superior se caracterizó por diversos rasgos: su implantación metropolitana; la centralidad hegemónica de una única universidad pública, el profesionalismo de la oferta académica; la descoordinación inter-institucional de las políticas públicas; la invisibilidad de otros circuitos -públicos o privados- de educación superior, rasgos que se completaron con una escasa oferta de personal académico con doctorado; la débil articulación con el sector productivo que en 2005 presentaba una marcada indigencia en materia de innovación. Finalmente, hay que apuntar la muy débil incorporación de entornos virtuales de aprendizaje, los déficits de internacionalización y la falta de decisiones en torno a un sistema de aseguramiento de la calidad.

Nos enfrentamos pues a un escenario "en prórroga" sine die de las reformas sistémicas relevantes, que dibujan el perfil de un sistema de educación superior asimétrico y en transición. (MARTÍNEZ LARRECHEA; CHIANCONE, 2011, p. 131-132). Ello se expresa a nivel macro en la ausencia de instancias legítimas y efectivas de planeamiento y de formulación de políticas al nivel del estado nacional como tal, que pudieran genera una imagen-objetivo y un conjunto convergente de actores y de políticas (como puede serlo el Consejo Nacional de Universidades en Argentina, o el Consejo Nacional de Educación en su Cámara de Educación Superior, en Brasil, entre múltiples otros ejemplos en los niveles latinoamericano y mundial.

El sistema carece de un marco normativo de rango legal, de una ley de educación superior. En el terreno normativo, subsiste un esquema laxo y más o menos inconexo, que incluye la ley orgánica de la Universidad de la República; la ley de creación de la Universidad Tecnológica -UTEC; el decreto-ley de universidades privadas y sus decretos reglamentarios; una ley general de educación, con alusiones programáticas a la educación superior y una ley de educación militar y policial, aun sin reglamentación. Un proyecto de ley de creación de una posible Universidad de la Educación fracasó en alcanzar los consensos básicos. Falta una ley de educación superior que asigne un lugar a todos los actores en el marco de eventuales normativas sectoriales.

Un aspecto crucial de la transformación universitaria es la democratización del acceso, la permanencia, los aprendizajes de calidad y el egreso oportuno de la Enseñanza Media, lo que, a su turno, obliga a transformar el proceso de aprendizaje desde la enseñanza primaria. 
La omisión universitaria en trabajar con otros organismos del Estado -durante todo el período 1985-2005- en la transformación de la educación básica, constituye uno de los principales elementos de sustentación de los actuales déficits educativos, ya que al bloquearse la eficiencia terminal del nivel medio y contenerse la expansión de la matrícula, se puso freno también a la diferenciación interna, a la internacionalización sustantiva y solidaria, y al dinamismo del sistema de educación superior.

La creación de una agencia de acreditación, que dio lugar a dos proyectos de ley, en 2007 y 2010, fue también neutralizada por la cultura de fragmentación y descoordinación del sistema, problemas de base a los que dicho proyecto era por completo indiferente. La lógica del control gubernamental y burocrático terminó por bloquear cualquier posible consenso al respecto.

Uruguay aparece como un adoptante tardío y problemático -también desactualizadode las políticas internacionales de aseguramiento de la calidad.

En los hechos, se fue consolidando un paradigma conservador en educación superior, que hoy solo aparece desafiado por algunas políticas puntuales (la descentralización universitaria; la creación de algunas posibles nuevas instituciones; y el establecimiento de una agencia implementadora, y a menudo formuladora de políticas de ciencia, tecnología e innovación (ANII).

En suma, mientras la matriz tradicional ha contribuido a la neutralización de los cambios centrales, crítico-estructurales y estratégicos, el proceso de los últimos veinticinco años es, de todas maneras, bastante rico en modificaciones relevantes, a nivel público y privado. Pero esto no ha sido suficiente para dar cuenta de una transformación sistémica.

En este contexto, las alternativas pasan por la adopción consensuada de un sistema de aseguramiento de la calidad que otorgue centralidad a un esquema de autorregulación, sostenido con fondos públicos, que permita desarrollar la cultura de la calidad de las instituciones, a la vez que faculte a los órdenes universitarios y a otros interesados clave en ese proceso. Y como principal dispositivo del esquema de autorregulación, se requiere el fortalecimiento de mecanismos y sistemas de garantía interna de la calidad (SIGC).

Estos diseños alternativos de política pueden favorecer a su vez un consenso nacional en torno a un texto legal, que se descentre de su reiterada obsesión por el control burocrático de la diferenciación institucional y académica, que supere la asimetría estructural y que potencie alternativas sistémicas, entre ellas la innovación y la coordinación en gran escala, la internacionalización y la adopción de estándares altos de calidad académica -sin limitar la riqueza de los proyectos académicos singulares). 
El futuro de la educación superior uruguaya depende de que los cambios, reales pero graduales y limitados que ha experimentado el sistema en el último cuarto de siglo, puedan articularse y potenciarse en un nuevo diseño institucional y en torno a un nuevo impulso de políticas públicas.

En el centro de esta nueva generación de políticas públicas deben estar la calidad y la equidad de la educación básica, la implantación nacional de la educación superior, el reconocimiento de la formación docente como parte de la educación superior universitaria, la regionalización e internacionalización solidarias y, desde luego un sistema de aseguramiento de la calidad que no intente dar las mismas respuestas de las décadas de 1990 y 2000.

En el fondo, lo que está en juego es la relación de la universidad con el conocimiento público, con su uso apropiado, racional y crítico, con su distribución social equitativa y con sus potencialidades de acompañar la transformación social, en un contexto caracterizado por la crisis del capitalismo global, del regionalismo y del imaginario humanista de raíz kantiana.

\section{Referencias}

\section{DÁVILA, M.; MARTÍNEZ LARRECHEA, Enrique. Evaluación y Acreditación en}

Argentina y Uruguay: los sistemas de educación superior y nuevas orientaciones de política en perspectiva comparada. (2009) Disponible en: <http://rlcu.org.ar/recursos/E_0000030_003 Argentina.pdf>. Acesso en:

DÁVILA, M., DIAZ MAYNARD, Á., FERNÁNDEZ, J. Et. al. La regulación de la Educación Superior Privada en Uruguay: análisis y discusión del Decreto 104/14. Debate Universitario, v.3, n.5, p.67-107. Disponible en: <http://ppct.caicyt.gov.ar/index.php/debateuniversitario/article/viewFile/5349/pdf>. Acesso en:

DIAS SOBRINHO, Jose. Avaliação e transformações da educação superior brasileira (19952009): do provão ao SINAES. Avaliação: Revista da Avaliação da Educação Superior, v.15, n.1, 2010.

SANTOS FILHO, Jose Camilo. Especificidade da universidade: implicações para avaliação institucional. Pro-Posições, Campinas, v.6, n.1 [16], p.9-21, mar. 1995.

HAUG, Guy. Políticas de aseguramiento de la calidad en la Educación Superior Europea. En: CINDA. Aseguramiento de la calidad en América Latina. Santiago de Chile: CINDA, 2012

KELLS, H.R., Autorregulación en la educación chilena. Consejo Nacional de Educación, Santiago, Chile. 1993.

LEMAITRE, María José: Zenteno, María del Pilar. En: CINDA. Aseguramiento de la Calidad en América Latina. Santiago de Chile: CINDA, 2012. 
MARTÍNEZ LARRECHEA, Enrique. La educación superior de Uruguay en transición. Inercias y horizontes de cambio. Montevideo, Uruguay, 2003, Fundación de Cultura Universitaria.

MARTÍNEZ LARRECHEA, Enrique; Chiancone, CHIANCONE Adriana. La educación superior en Iberoamérica. Informe nacional de Uruguay. CINDA, 2006. (Mimeo.)

MARTÍNEZ LARRECHEA, Enrique; Chiancone, CHIANCONE Adriana. La Educación Superior en Uruguay: matriz inercial y escenarios de cambio. Revista de innovación educativa: Educación Superior en América Latina, Ciudad de México, v. 11, n. 57, oct./dic. 2011.

MARTÍNEZ LARRECHEA, Enrique. Las políticas públicas de evaluación y acreditación institucional en educación superior: una comparación de Argentina y Venezuela. Tesis de Maestría presentada a FLACSO Argentina, 2000. Buenos Aires: 2000. (Mimeo.) 